STRUCTURAL SCIENCE CRYSTAL ENGINEERING MATERIALS

ISSN 2052-5206

Received 2 August 2018

Accepted 14 November 2018

Edited by M. de Boissieu, SIMaP, France

Keywords: cobalt ferrite; spinel structure; epitaxial growth; surface $\mathrm{X}$-ray diffraction; resonant $\mathrm{X}$-ray diffraction.

Supporting information: this article has supporting information at journals.iucr.org/b

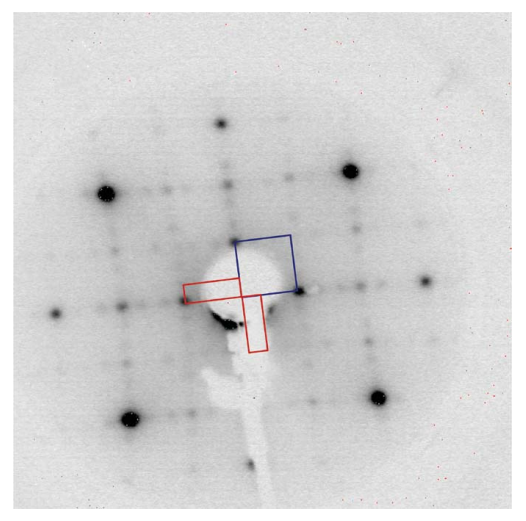

C 2019 International Union of Crystallography

\section{Epitaxial growth and structure of cobalt ferrite thin films with large inversion parameter on $\mathrm{Ag}(001)$}

\author{
Maurizio De Santis, ${ }^{a *}$ Aude Bailly, ${ }^{a}$ Ian Coates, ${ }^{a}$ Stéphane Grenier, ${ }^{a}$ \\ Olivier Heckmann, ${ }^{\mathrm{b}}$ Karol Hricovini, ${ }^{\mathrm{b}}$ Yves Joly, ${ }^{\mathrm{a}}$ Véronique Langlais, \\ Aline Y. Ramos, ${ }^{a}$ Christine Richter, ${ }^{\text {b }}$ Xavier Torrelles, ${ }^{d}$ Stéphanie Garaudée, ${ }^{\text {a }}$ \\ Olivier Geaymond ${ }^{\mathrm{a}}$ and Olivier Ulrich ${ }^{\mathrm{e}}$
}

anstitut Néel, Université Grenoble Alpes, CNRS, Grenoble INP, Grenoble 38042, France, 'bMPS, Université de CergyPontoise, Neuville/Oise, Cergy-Pontoise 95031, France, 'CEMES, CNRS, BP 94347, 29 rue Jeanne Marvig, Toulouse 31055, France, ' Institut de Ciència de Materials de Barcelona (ICMAB), CSIC, Bellaterra, Barcelona 08193, Spain, and e INAC/MEM, Université Grenoble Alpes, CEA, Grenoble 38054, France. *Correspondence e-mail:

maurizio.de-santis@neel.cnrs.fr

Cobalt ferrite ultrathin films with the inverse spinel structure are among the best candidates for spin filtering at room temperature. High-quality epitaxial $\mathrm{CoFe}_{2} \mathrm{O}_{4}$ films about $4 \mathrm{~nm}$ thick have been fabricated on $\mathrm{Ag}(001)$ following a three-step method: an ultrathin metallic $\mathrm{CoFe}_{2}$ alloy was first grown in coherent epitaxy on the substrate and then treated twice with $\mathrm{O}_{2}$, first at room temperature and then during annealing. The epitaxial orientation and the surface, interface and film structure were resolved using a combination of lowenergy electron diffraction, scanning tunnelling microscopy, Auger electron spectroscopy and in situ grazing-incidence X-ray diffraction. A slight tetragonal distortion was observed, which should drive the easy magnetization axis in-plane due to the large magneto-elastic coupling of such a material. The so-called inversion parameter, i.e. the Co fraction occupying octahedral sites in the ferrite spinel structure, is a key element for its spin-dependent electronic gap. It was obtained through in situ resonant X-ray diffraction measurements collected at both the Co and $\mathrm{Fe} K$ edges. The data analysis was performed using FDMNES, an $a b$ initio program already extensively used to simulate X-ray absorption spectroscopy, and shows that the Co ions are predominantly located on octahedral sites with an inversion parameter of 0.88(5). Ex situ X-ray photoelectron spectroscopy gives an estimation in accordance with the values obtained through diffraction analysis.

\section{Introduction}

Cobalt ferrite is an insulating (ferri)magnetic oxide with a high Curie temperature $\left(T_{\mathrm{C}}=793 \mathrm{~K}\right)$ and a large saturation magnetization (Brabers, 1995). Alongside its low cost, these properties make it attractive for a wide range of applications. Its spinel structure (space group $F d \overline{3} m$ ) comprises a distorted face-centred cubic (f.c.c.) sublattice of $\mathrm{O}^{2-}$ anions in which one eighth of the tetrahedral lattice holes and one half of the octahedral lattice holes are occupied by cations $\left(\mathrm{Fe}^{3+}\right.$ or $\left.\mathrm{Co}^{2+}\right)$. This results in the general formula $A B_{2} \mathrm{O}_{4}$, where $A$ and $B$ refer to the cations located on the tetrahedral and octahedral sites, respectively. In the normal spinel structure, the $A$ sites are occupied by divalent cations and the $B$ sites by trivalent cations. In the inverse spinel structure, the divalent cations occupy half of the $B$ sites and the trivalent cations occupy the remaining $A$ and $B$ sites (Proskurina et al., 2004). For cobalt ferrite, the inverse structure is the most stable. However, in general, this inversion is not complete and a fraction of the Co 
ions remain located on tetrahedral sites. The degree of inversion typically depends on the sample preparation conditions. Due to ferromagnetic interactions between the ions on the octahedral sites, and antiferromagnetic interactions between the ions on the octahedral and tetrahedral sites, cobalt ferrite is ferrimagnetic. Various density-of-state (DOS) calculations have accordingly predicted that the electronic band gap at the Fermi level differs for majority and minority spins (Fritsch \& Ederer, 2010; Caffrey et al., 2013; Szotek et al., 2006), while the size of the band gap depends on the degree of inversion (Fritsch \& Ederer, 2011).

The use of ferromagnetic or ferrimagnetic insulators in multilayered structures is an efficient way to generate highly spin-polarized currents due to the exponential relationship between tunnelling probability and the spin-dependent barrier height. This so-called spin-filtering effect was first observed in EuS at low temperature (Moodera et al., 1988), but in the last decade research has focused on ferrites because of their high Curie temperatures, giving the possibility of spin-filter tunnelling at room temperature. A second important property of cobalt ferrite is its significant magnetostriction (Bozorth $e t$ al., 1955) that results in a large strain-dependent magnetocrystalline anisotropy energy in ultrathin epitaxial films. A compressive strain favours an in-plane magnetization, as in the case of $\mathrm{CoFe}_{2} \mathrm{O}_{4} / \mathrm{MgAl}_{2} \mathrm{O}_{4}(001)$ (Matzen et al., 2011), while tensile strain induces a perpendicular magnetization, observed for $\mathrm{CoFe}_{2} \mathrm{O}_{4} / \mathrm{MgO}(001)$ (Chambers et al., 2002; Lisfi \& Williams, 2003; Yanagihara et al., 2011). These findings have been confirmed by theoretical calculations (Fritsch \& Ederer, 2010). Therefore, the incorporation of cobalt ferrite in artificial multiferroic heterostructures may result in new phenomena that could open the way to a large range of applications. It has been shown, for example, that an elastic strain-mediated magnetoelectric coupling can be used to reverse the magnetization in columnar $\mathrm{CoFe}_{2} \mathrm{O}_{4}$ nanostructures embedded in ferroelectric $\mathrm{BiFeO}_{3}$ (Zavaliche et al., 2005).

To fine tune the inversion parameter and induce epitaxial strain, a precise growth methodology is essential. The growth of transition metal oxides on f.c.c. (001) metallic substrates is strongly influenced by the lattice misfit. For example, $\mathrm{CoO}$ films grow (001) oriented on $\mathrm{Ag}(001)$ (Torelli et al., 2007), while in the case of $\mathrm{CoO} / \operatorname{Ir}(100)$, (111) films are usually obtained (Meyer et al., 2008). However, in this latter system the $\mathrm{CoO}$ orientation can be changed to (001) by depositing a Co buffer layer about two monolayers (ML) thick prior to oxidation (Gubo et al., 2012). This layer is pseudomorphic and forms, after moderate oxidation, a $c(4 \times 2)-\mathrm{Co}_{3} \mathrm{O}_{4} / \mathrm{Co} / \mathrm{Ir}(001)$ reconstructed surface, which acts as a precursor for the growth of $\mathrm{CoO}(001)$. A similar method for growing high-quality (001) magnetite ultrathin films on $\operatorname{Ag}(001)$ has already been demonstrated (Lamirand et al., 2016). Facilitated by a lattice mismatch of only $0.8 \%$, Fe grows pseudomorphically on $\mathrm{Ag}(001)$. Provided that a few ML of Fe are initially deposited, the lattice expands during oxidation, but its relative orientation is maintained. Here, the same technique is employed to obtain high-quality ultrathin cobalt ferrite films with a sharp interface, a relatively flat surface and a large inversion parameter.

In the next section, the experimental setups and deposition methods are described, together with a qualitative characterization of the surface. In Section 3, the film structure is solved by grazing-incidence X-ray diffraction (GIXRD). Sections 4 and 5 are devoted to the determination of the inversion parameter, by X-ray photoelectron spectroscopy (XPS) and resonant X-ray diffraction (RXD), respectively.

\section{Setups, sample growth and characterization}

All films were prepared in a similar manner using one of two distinct experimental setups, both of which are fully equipped for sample preparation and analysis in an ultra-high vacuum (UHV) environment. The first setup uses low-energy electron diffractometry (LEED), scanning tunnelling microscopy (STM) and Auger electron spectrometry (AES), and it is located at the Néel Institute. Samples grown at this location were then transferred to the LMPS laboratory in CergyPontoise, where photoelectron spectra were measured using an $\mathrm{Mg} K \alpha \mathrm{X}$-ray emission source $(1253.6 \mathrm{eV})$ and a hemispherical analyser. The second setup (GIXRD) is installed on the French BM32 beamline (CRG-IF) at the European Synchrotron Radiation Facility (ESRF, Grenoble, France). This setup consists of a UHV chamber equipped with evaporation sources for molecular-beam epitaxy (MBE) growth and with an Auger electron spectrometer. The system is mounted on a $Z$-axis diffractometer, with additional degrees of freedom for sample positioning provided by a hexapod. This setup was used for resonant and non-resonant X-ray diffraction experiments in situ.

The oxide layers were grown on an $\operatorname{Ag}(001)$ single crystal with a miscut of less than $0.1^{\circ}$. Prior to deposition, the substrate was cleaned by repeated cycles of $\mathrm{Ar}^{+}$ion sputtering, followed by annealing at approximately $850 \mathrm{~K}$. Cleanliness was checked by AES, such that all contaminants were below the detection limit. Iron and cobalt were evaporated from pure rods using water-cooled electron-beam evaporators. The base pressure was in the low $10^{-11}\left(10^{-10}\right)$ mbar range for the STM (GIXRD) setup $(1 \mathrm{bar}=100000 \mathrm{~Pa})$. The Fe $(\mathrm{Co})$ deposition rate was typically $1 \mathrm{ML}$ every $5 \mathrm{~min}(10 \mathrm{~min})$, calibrated with a quartz crystal microbalance. STM images were obtained in constant-current mode using a voltage bias $\left(V_{\text {sample }}\right)$ applied to the sample. The non-resonant GIXRD measurements were performed with a photon energy of $9500 \mathrm{eV}$. Resonant measurements were carried out at both Co and Fe $K$ absorption edges. To increase the signal-to-noise ratio, the incidence angle was set at the critical angle for total $\mathrm{X}$-ray reflection for $\mathrm{Ag}$ at the different energies $(0.37,0.46$ and $0.50^{\circ}$ for 9500,7709 and $7112 \mathrm{eV}$, respectively). The diffraction data were collected using a 2D detector (MAXIPIX, ESRF).

A cobalt ferrite seed layer was initially prepared in the LEED/STM setup by a three-step method. Firstly, Co (2 ML as referred to the Ag surface atomic density) and $\mathrm{Fe}$ (4 ML) were codeposited on the substrate kept at room temperature (RT; 350-300 K) under UHV, forming an epitaxial metallic 
alloy. After deposition, the oxide layer was formed by dosing with $10^{-6}$ mbar $\mathrm{O}_{2}$ for $10 \mathrm{~min}$ at RT. This step is essential to avoid intermixing with $\mathrm{Ag}$. Finally, the $\mathrm{O}_{2}$ partial pressure was maintained whilst the sample was annealed up to $750 \mathrm{~K}$ for $10 \mathrm{~min}$ via an intermediate $10 \mathrm{~min}$ interval at $570 \mathrm{~K}$. Following the deposition of the seed layer, the film thickness was increased by reactive co-deposition of $\mathrm{Co}$ and $\mathrm{Fe}$ ( $2 \mathrm{ML}$ and $4 \mathrm{ML}$, respectively) in the presence of molecular oxygen $\left(10^{-6}\right.$ mbar) at $750 \mathrm{~K}$, resulting in a cobalt ferrite film about $4 \mathrm{~nm}$ thick. For the GIXRD experiments, the samples were grown following the same procedure, with the exception that oxygen annealing was performed up to a higher temperature of $\sim 870 \mathrm{~K}$ with more temperature intervals during the heating process. During annealing, the film structure was monitored at each interval. The higher temperature was also maintained during the reactive deposition, which subsequently ensured a large average crystallite domain size in the surface plane: a value of about $30 \mathrm{~nm}$ was found, applying the Scherrer equation to the ferrite peak width. This allows a proper measurement of the diffraction rod intensity without the need for corrections of the active detector area. A large domain size is also crucial for the establishment of bulk-like ferrite properties. It is known that the presence of antiphase domains in ultrathin magnetite films can result in superparamagnetic behaviour (Voogt et al., 1998).

Fig. 1 shows AES spectra measured in the LEED/STM setup (continuous red line) and in the GIXRD one (black circles) after oxide growth. The principal peaks of $\mathrm{O}, \mathrm{Fe}$ and $\mathrm{Co}$ are labelled. The peaks at 598 and $775 \mathrm{eV}$, arising from Fe and Co levels, respectively, were used to infer the ferrite stoichiometry. The two samples have the same composition, within

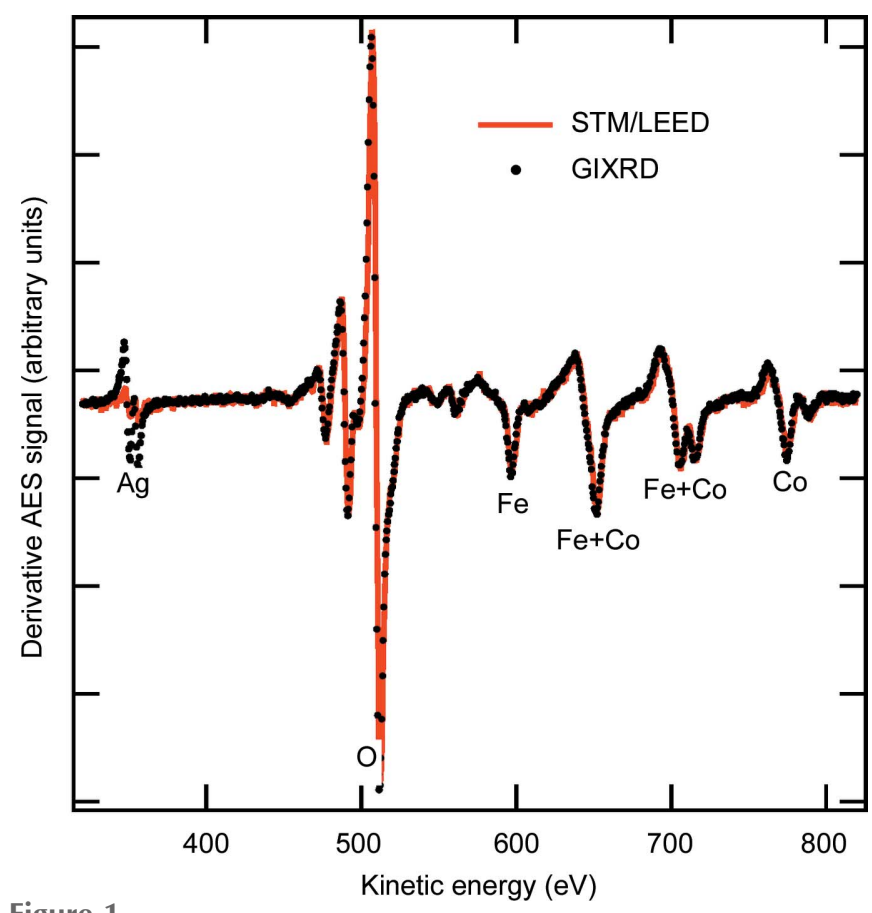

Figure 1

Derivative Auger spectra of the samples elaborated in the GIXRD chamber (black circles) and in the LEED/STM chamber (continuous red line). a)

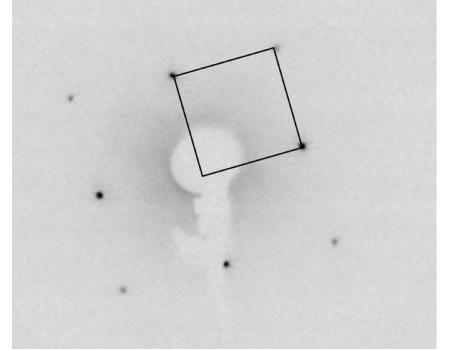

Figure 2

LEED patterns of (a) clean $\mathrm{Ag}(001)$ and $(b) \mathrm{CoFe}_{2} \mathrm{O}_{4} / \mathrm{Ag}(001)$. Both were recorded with an electron-beam energy of $75 \mathrm{eV}$. The ferrite film grows $(001)$ oriented and exhibits a $(3 \times 1)$ surface reconstruction. The $\mathrm{Ag}$, ferrite and $(3 \times 1)$ reciprocal-space surface unit cells are represented by the black square, blue square and red rectangles, respectively.

error. We measure a signal ratio $\mathrm{AES}\left(\mathrm{Fe}_{598}\right) / \mathrm{AES}\left(\mathrm{Co}_{775}\right)$ of $\sim 0.75$, while the corresponding cross-section ratio is $\sigma\left(\mathrm{Fe}_{598}\right) /$ $\sigma\left(\mathrm{Co}_{775}\right) \simeq 0.46$. Then the ratio $\mathrm{Fe}: \mathrm{Co} \simeq 1.63$, i.e. the ferrite is slightly enriched in Co with respect to the desired composition. As will be discussed later, the more quantitative X-ray resonant diffraction analysis gives a ratio of $\sim 1.86$. Some $\mathrm{Ag}$ surface segregation is observed in the sample annealed at $870 \mathrm{~K}$ (peak at $356 \mathrm{eV}$ ). A rough estimation based on the relative cross sections gives about 0.5 equivalent $\mathrm{ML}$ of $\mathrm{Ag}$ on the surface.

Fig. 2 shows the LEED pattern of ( $a$ ) the Ag substrate and (b) the final oxide, both measured with an electron-beam energy of $75 \mathrm{eV}$. The latter is generated by an epitaxial oxide layer with $P 4 \mathrm{~mm}$ symmetry and a unit-cell constant almost

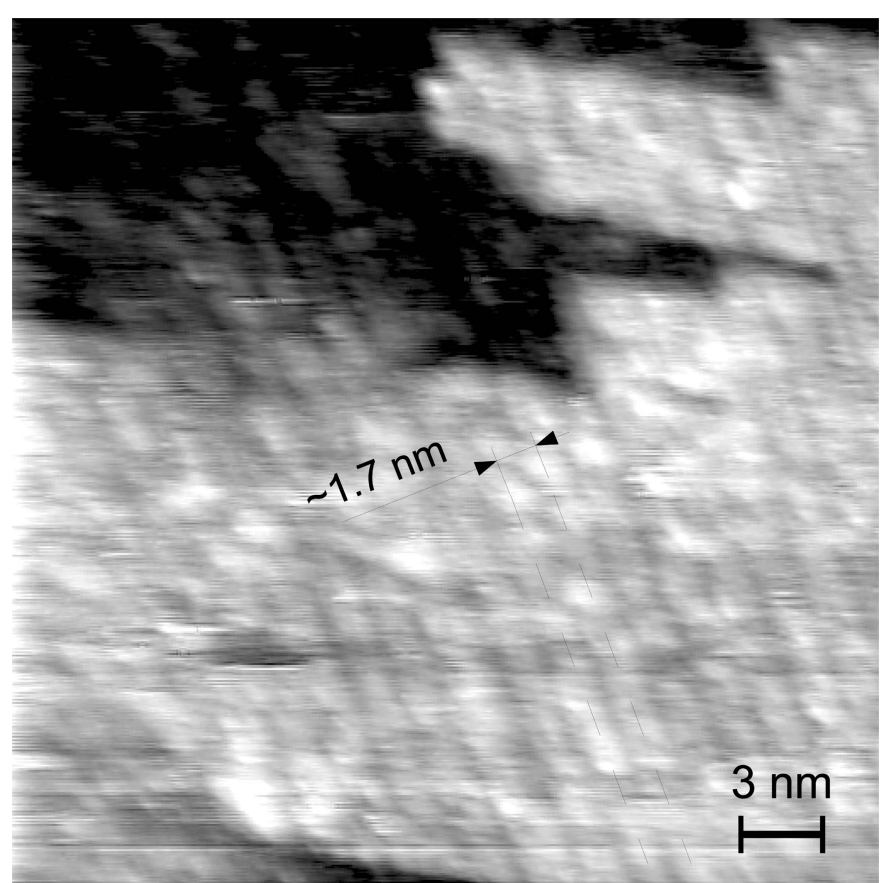

Figure 3

An STM image of the cobalt ferrite ultrathin film recorded at room temperature $\left(30 \times 30 \mathrm{~nm}, V_{\text {sample }}=2 \mathrm{~V}, I_{\text {bias }}=0.1 \mathrm{nA}\right)$. 
twice that of $\mathrm{Ag}$. This fits well with an (001)-oriented $\mathrm{CoFe}_{2} \mathrm{O}_{4}$ film. In addition, weaker spots of two domains at $90^{\circ}$ of a $(3 \times 1)$ surface reconstruction are observed.

A $30 \times 30 \mathrm{~nm}$ medium-resolution STM image of the same oxide sample recorded at room temperature is shown in Fig. 3. The surface is flat on such a length scale and rows spaced by about $1.7 \mathrm{~nm}$, the period of the $(3 \times 1)$ reconstruction, are

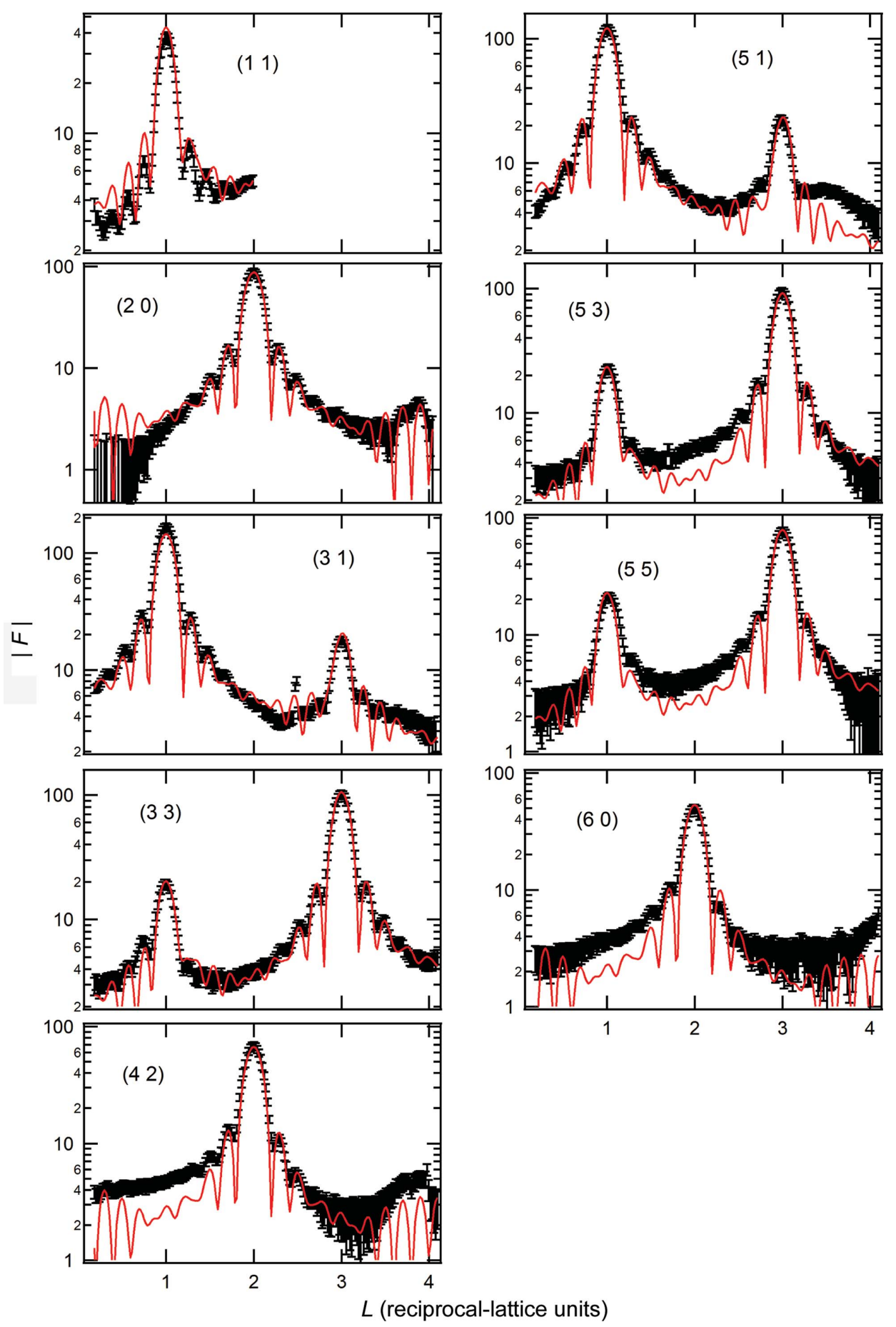

Figure 4

Experimental $\mathrm{CoFe}_{2} \mathrm{O}_{4}$ film structure factors measured along nine inequivalent rods with relative error bars (black symbols) and best fits (continuous red lines). The corresponding $(H K)$ values are indicated on each panel. $L$ is related to the $c_{\text {film }}$ unit-cell constant. clearly observed. The step height between dark and clear regions in the figure is about $0.22(3) \mathrm{nm}$. Large size images show that this value is the most often encountered as the step height (while scanning with $V_{\text {sample }}=2 \mathrm{~V}$ ).

The ferrite structure can be viewed along the (001) direction as a stacking of eight unique layers that alternate between planes containing oxygen anions and $B$-site cations, and planes containing only $A$-site cations. The interplanar spacing between planes of the same type is $0.21 \mathrm{~nm}$, which is directly comparable with the observed step height. It can therefore be concluded that the surface termination of the cobalt ferrite structure is dominated by one of these two types of plane. A similar result was observed at the surface of (001) bulk magnetite, which exhibits $B$-type termination (Stanka et al., 2000).

\section{GIXRD}

GIXRD data were collected to solve the structure quantitatively. The diffraction pattern has four-fold symmetry, as already observed by LEED. The in-plane [100] direction is aligned with the [100] one of the silver substrate, indicating epitaxial growth. The film is relaxed and its diffraction pattern does not interfere with that of the substrate. Two sets of sharp peaks are therefore observed while scanning the momentum transfer, $\mathbf{Q}$, parallel to the surface $[Q=(4 \pi / \lambda) \sin (\theta / 2)$, where $\theta$ is the scattering angle and $\lambda$ is the wavelength of the incident radiation]. The first set is given by the so-called crystal truncation rods (CTRs) of Ag (Robinson, 1986), while the second set corresponds to the rods originating from the film. They are located at integer $(H K)$ values, once indexed in the film mesh reference. Perpendicular to the surface, an intensity distribution, the so-called diffraction rod, is observed for each $(H K)$ value (Robinson, 1991). It exhibits wide peaks and thickness intensity oscillations.

The in-plane film unit-cell constant $a_{\text {film }}$ was obtained by scanning across some diffraction rods at $Q_{z}=2 \pi / a_{\text {cfo }}$, where $a_{\text {cfo }}$, the 
bulk cobalt ferrite unit-cell constant, is 838.6 pm (Proskurina et al., 2004; Mohamed \& Yehia, 2014). The positions of six rods (including five non-equivalent ones) were measured carefully. A linear regression analysis gives $a_{\mathrm{film}}=836(2) \mathrm{pm}$. With the objective of achieving a representative portion of reciprocal space, a large set of intensities along several rods, $I_{H K}(L)$, was measured. The standard procedure is to set the diffractometer angles to define each $(H K L)$ point in the sample's reciprocal space and then rock the sample azimuth to integrate the intensity across the rod at a given $L$ value. The structure factors $F_{H K L}$ are then extracted by applying standard correction factors (Vlieg, 1997). A set of 416 reflections distributed along 14 rods was collected. The set averaged to 293 nonequivalent reflections according to the substrate's $P 4 \mathrm{~mm}$ surface symmetry (ten non-equivalent rods). An agreement factor of $7 \%$ between the structure factors of equivalent reflections was found and used as a systematic error estimation for the final experimental error calculation (Robinson, 1991).

The sampling interval required to describe the rod shape, $\Delta Q_{z}$, decreases with respect to the inverse of the film thickness, and the required acquisition time increases accordingly. In this study, an initial $\Delta Q_{z}$ value of $0.1 \times 2 \pi / a_{\text {cfo }}$ was used, which proved to be insufficient to describe the film thickness oscillations. Subsequently, line scans along each rod were collected (stationary or $L$ scans, $\Delta Q_{z}=0.02 \times 2 \pi / a_{\text {cfo }}$ ) to which specific corrections were applied to extract the structure factors. These $F_{H K L}$ curves were interpolated and multiplied by a specific scaling factor to fit the data obtained from the rocking scans. The resulting rods are plotted in Fig. 4. The $Q_{z}$ values have been renormalized using a factor calculated from the cobalt ferrite density, to take into account the X-ray refraction of the incident beam at the vacuum-film interface (Feidenhans'l, 1989).

The first step in the analysis was the calculation of the average film interlayer spacing by fitting the peak positions along the rods. Using a linear regression based on the 14 nonequivalent peaks, a $c_{\text {film }}$ value of 841 (3) pm was found, resulting in a slight tetragonal distortion whereby $c_{\mathrm{film}} / a_{\mathrm{film}}=$
1.006 (6). The unit-cell volume was found to be equal to that of the bulk material, within error, $\left(V_{\text {film }}-V_{\text {cfo }}\right) / V_{\text {cfo }}=$ $-2(8) \times 10^{-3}$.

Before resolving the film structure in detail, the interface roughness, which modifies the shape of the rods, was studied by analysing the Ag CTRs. A set of 47 reflections belonging to three different CTRs was collected. Their structure factors are reported in Fig. 5, indexed within the $\operatorname{Ag}(001)$ surface cell $\left(\mathbf{a}_{s}^{1}\right.$ $=\frac{a_{\mathrm{Ag}}}{2}[1 \overline{10} 0], \mathbf{a}_{s}^{2}=\frac{a_{\mathrm{Ag}}}{2}[110]$ and $\left.\mathbf{a}_{s}^{3}=a_{\mathrm{Ag}}[001]\right)$. The error is evaluated using the agreement factor obtained from the film reflections.

Since the oxide film exists in incoherent epitaxy, it does not contribute to the CTR intensity, which depends only on the substrate parameters and the interface roughness. An initial best fit of the (11) CTR is shown in Fig. 5(a) (dashed red line). It is calculated using bulk interlayer distances and DebyeWaller (DW) factors $\left(B_{\mathrm{Ag}}=0.7 \times 10^{4} \mathrm{pm}^{2}\right)$. The roughness, considered within the $\beta$ model (Robinson, 1986), is therefore the only remaining physical parameter to be optimized. A value of $\beta=0.12$ (3) is obtained, which corresponds to a rootmean-square (r.m.s.) roughness of $80 \mathrm{pm}$. This represents quite a sharp interface. The resulting normalized $\chi^{2}$ is 0.84 .

However, as shown in Fig. 5, this simple model fails to give an accurate fit to the relative intensity of the different rods. The $\chi^{2}$ increases to about 10 upon considering the full data set of three CTRs. A better description is achieved by considering the role of the oxide film. The stress exerted by the film on the $\mathrm{Ag}$ at the interface induces a localized displacement field in the Ag substrate. Periodic displacements in the substrate give rise to satellite peaks close to the Bragg ones (Prévot et al., 2007). The presence of these satellites is discussed in the supporting information.

The stress caused by the oxide film induces displacements that are predominantly parallel to the interface. Their amplitude decreases while going deeper into the crystal. A detailed analysis of the displacement field would require the quantitative measurement of satellite rods. However, the average structure can be described by CTR analysis as a structural

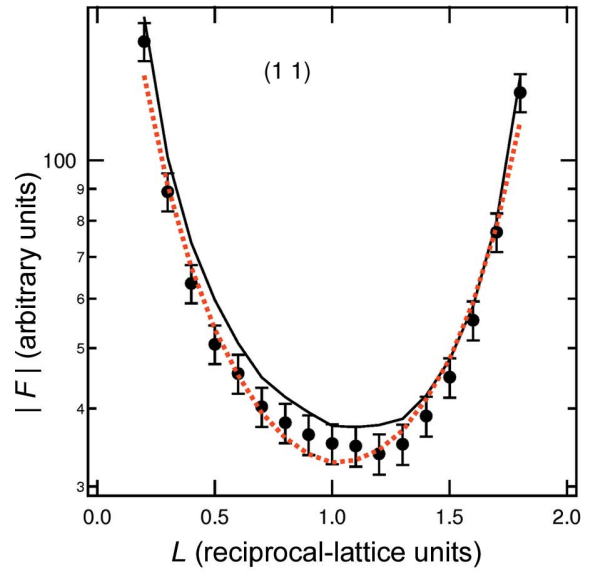

(a)

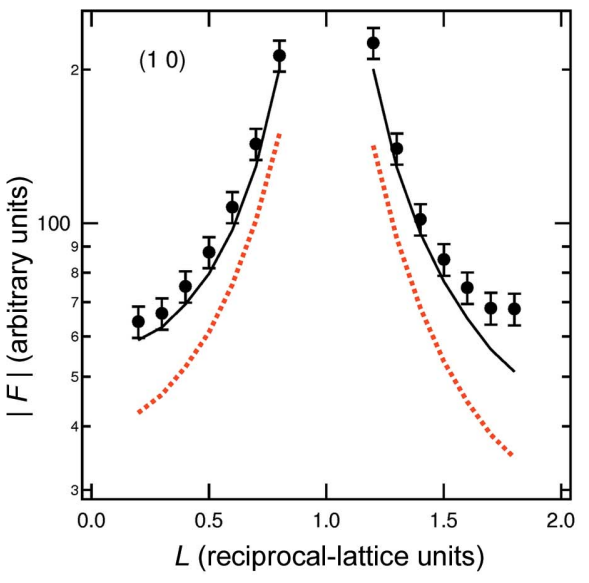

(b)

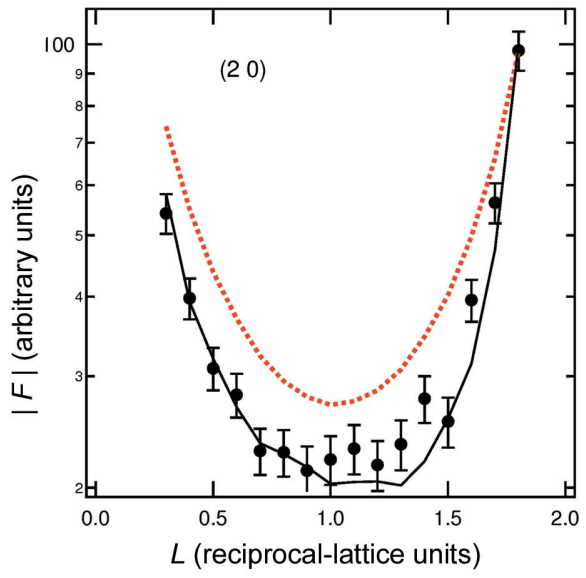

(c)

Figure 5

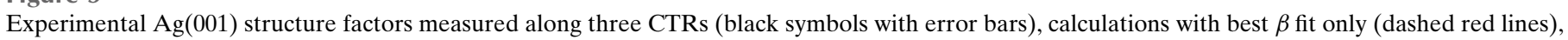
and final fits performed optimizing five in-plane surface DW factors and the first two Ag interlayer distances (solid black lines). 
Table 1

Best-fit structural parameters for interfacial Ag compared with values for clean $\mathrm{Ag}(100)$.

Including the interlayer distances $d_{12}$ and $d_{23}$, the DW factor at the $i$ th layer parallel to the surface, $B_{i, \mathrm{IP}}$, and the roughness parameter $\beta$.

\begin{tabular}{lcc}
\hline & This work & Clean $\operatorname{Ag}(100) \dagger$ \\
\hline$\Delta d_{12} / d_{\text {bulk }}(\%)$ & $-1.5(8)$ & $-0.8(8)$ \\
$\Delta d_{23} / d_{\text {bulk }}(\%)$ & $0.5(3)$ & $1.0(8)$ \\
$B_{1, \mathrm{IP}}\left(\times 10^{4} \mathrm{pm}^{2}\right)$ & $6.3(5)$ & 0.95 \\
$B_{2, \mathrm{IP}}\left(\times 10^{4} \mathrm{pm}^{2}\right)$ & $4.0(5)$ & 0.95 \\
$B_{3, \mathrm{IP}}\left(\times 10^{4} \mathrm{pm}^{2}\right)$ & $2.4(3)$ & \\
$B_{4, \mathrm{IP}}\left(\times 10^{4} \mathrm{pm}^{2}\right)$ & $1.4(3)$ & \\
$B_{5, \mathrm{IP}}\left(\times 10^{4} \mathrm{pm}^{2}\right)$ & $0.9(1)$ & \\
$\beta$ & $0.05(3)$ & \\
$\chi^{2}$ & 2.5 & \\
\hline
\end{tabular}

$\dagger$ Meyerheim et al. (1997).

disorder within the Ag surface unit cell. The CTRs were therefore analysed further by introducing in-plane DW factors. Simultaneous fits of the CTRs (Fig. 5, continuous black lines) result in an in-plane DW factor gradient over the first five atomic layers of the Ag substrate at the interface $\left(B_{i, \mathrm{IP}}\right)$. The out-of-plane component was kept fixed at the bulk value. Note that the DW factors observed in the surface layers decrease the scattering amplitude relative to the bulk value, therefore their effect on a single rod is similar to that of increasing interface roughness. Within this model, the best-fit $\beta$ value is close to zero. Clean $\mathrm{Ag}(001)$, as well as several other metals, exhibit an oscillating variation in the interlayer distance close to the surface (Meyerheim et al., 1997). The two Ag interlayer distances closest to the interface, $d_{12}$ and $d_{23}$, were therefore optimized. It was found that the former is contracted by $1.5 \%$, while the latter increases slightly. The corresponding $\chi^{2}$ when considering the full data set of the three CTRs is 2.5. The best-fit values are reported in Table 1.

In summary, the CTR analysis shows that the interface is quite sharp, despite the high annealing temperature, as indi-

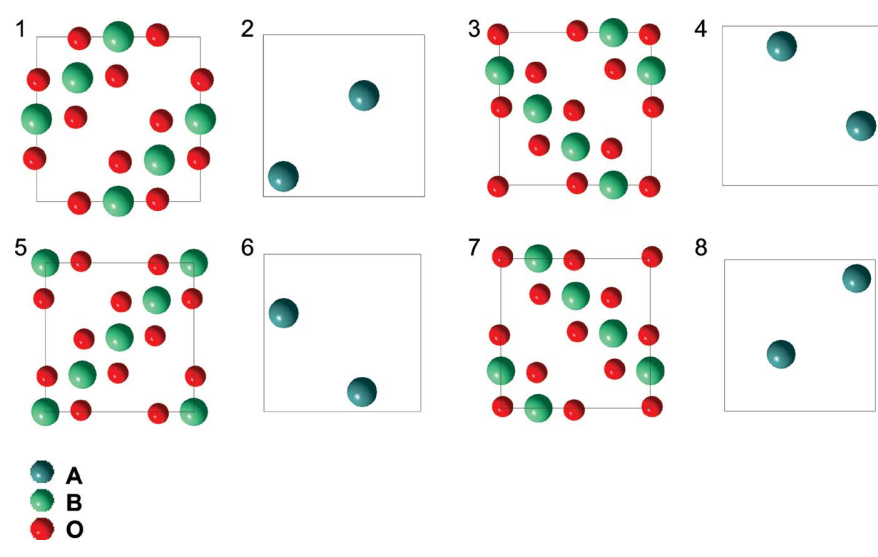

Figure 6

The layer-by-layer structure of the bulk $\mathrm{CoFe}_{2} \mathrm{O}_{4}$ unit cell, following the stacking order. $A$ and $B$ correspond to the tetrahedral and octahedral sites, respectively. The interlayer spacing is $c_{\text {film }} / 8$.
Table 2

Experimental and fitted structure factors for integer $(H K L)$ values.

\begin{tabular}{lcr}
\hline Reflection index & Experimental & Fit \\
\hline$(111)$ & $38(3)$ & 41.8 \\
$(202)$ & $89(7)$ & 84.8 \\
$(311)$ & $168(12)$ & 140.8 \\
$(313)$ & $18(1)$ & 19.7 \\
$(331)$ & $19(1)$ & 19.7 \\
$(333)$ & $102(7)$ & 102.5 \\
$(422)$ & $69(5)$ & 64.8 \\
$(511)$ & $120(9)$ & 117.6 \\
$(513)$ & $22(2)$ & 22.7 \\
$(531)$ & $23(2)$ & 22.7 \\
$(533)$ & $94(7)$ & 88.9 \\
$(551)$ & $21(2)$ & 22.0 \\
$(553)$ & $77(6)$ & 76.8 \\
$(602)$ & $50(4)$ & 51.6 \\
$\chi^{2}$ & & 1.1 \\
\hline
\end{tabular}

cated by the calculated $\beta$ parameter of 0.05 , which corresponds to an r.m.s. roughness of 48 pm (Robinson, 1986). In the following, the film structure is studied via analysis of its rods, considering a sharp interface.

The bulk $\mathrm{CoFe}_{2} \mathrm{O}_{4}$ unit cell is formed by eight equi-spaced atomic layers along the [001] axis, as shown in Fig. 6. The diffraction pattern of the film was calculated for a structure which follows this same layer stacking, starting with a $B$ type layer at the interface (layer 1 ). Since the non-resonant $\mathrm{Fe}$ and Co atomic form factors are close in magnitude, standard diffraction methods cannot give reliable values for the film's inversion parameter, i.e. the relative $\mathrm{Co}$ and $\mathrm{Fe}$ occupancies of tetrahedral and octahedral sites $\left(x_{A, \mathrm{Co}}, x_{A, \mathrm{Fe}}, x_{B, \mathrm{Co}}\right.$ and $x_{B, \mathrm{Fe}}$, respectively). These values were obtained by RXD, as described below, and are used here in the best fit of the structure. The scattering factors of $\mathrm{Fe}, \mathrm{Co}$ and $\mathrm{O}$ ions were used. In the first step only 14 structure factors at integer $(H K L)$ were fitted using a bulk-like unit cell. In this case the only structural parameters are the shift in the oxygen anion positions $\Delta$, the oxygen DW factor $\left(B_{\mathrm{O}}\right)$ and the cation one $\left(B_{\text {cat }}\right.$, taken to be the same for $\mathrm{Co}^{2+}$ and $\left.\mathrm{Fe}^{3+}\right)$. Here, $\Delta=$ $\pm(0.25-u)$ is the shift (in cell units) relative to a regular f.c.c. sublattice in accordance with the $F d \overline{3} m$ symmetry. The parameter $u$ is 0.261 (Proskurina et al., 2004) or 0.253 (Mohamed \& Yehia, 2014) for the bulk material. Table 2 shows the best fits for the structure factors, resulting in a $\chi^{2}$ of 1.1 , which definitively confirms the $\mathrm{CoFe}_{2} \mathrm{O}_{4}$ structure of the film. The structural parameters of the best fit are reported in Table 3. Finally, Table 4 shows comparisons between the best fit and the experimental bulk unit-cell constants and cation-oxygen distances $d_{A \mathrm{O}}$ and $d_{B \mathrm{O}}$.

The thickness and roughness of the film were obtained from the refinement agreement between experimental and calculated structure factors along all the rods, with $u, B_{\text {cat }}$ and $B_{\mathrm{O}}$ fixed. In our model the film is composed of 33 complete atomic layers, on top of which the surface is terminated by partially occupied bilayers, corresponding to the observed surface roughness. Each bilayer was assumed to be $B$ terminated, in accordance with the observed termination of the magnetite (001) surface (Pentcheva et al., 2005; Bliem, McDermott et al., 
Table 3

Best-fit structural parameters for the $\mathrm{CoFe}_{2} \mathrm{O}_{4}$ film.

\begin{tabular}{ll}
\hline$x_{A, \mathrm{Co}}$ & $0.13(5)$ \\
$x_{A, \mathrm{Fe}}$ & $0.87(5)$ \\
$x_{B, \mathrm{Co}}$ & $0.46(3)$ \\
$x_{B, \mathrm{Fe}}$ & $0.54(3)$ \\
$u$ & $0.256(2)$ \\
$B_{\mathrm{cat}}\left(\times 10^{4} \mathrm{pm}^{2}\right)$ & $1.1(5)$ \\
$B_{\mathrm{O}}\left(\times 10^{4} \mathrm{pm}^{2}\right)$ & $3(2)$ \\
$\mathrm{Occ}_{1}$ & $0.95(5)$ \\
$\mathrm{Occ}_{2}$ & $0.6(1)$ \\
$\mathrm{Occ}_{3}$ & $0.6(1)$ \\
$\mathrm{Occ}_{4}$ & $0.2(1)$ \\
$\mathrm{Occ}_{5}$ & $0.2(1)$ \\
$d_{1}(\mathrm{pm})$ & $92(2)$ \\
$\mathrm{Occ}(B)$ & $1+0 /-0.05$ \\
\hline
\end{tabular}

Table 4

Best-fit unit-cell constants and interatomic distances compared with bulk values.

\begin{tabular}{lccc}
\hline & Best fit & Bulk $\dagger$ & Bulk \\
\hline$a(\mathrm{pm})$ & $836(2)$ & 838.6 & 838.6 \\
$c(\mathrm{pm})$ & $841(3)$ & & \\
$d_{A \mathrm{O}}(\mathrm{pm})$ & $190(2)$ & 197.5 & 186.0 \\
$d_{B \mathrm{O}}(\mathrm{pm})$ & $204(2)$ & 200.8 & 207.1 \\
\hline
\end{tabular}

$\dagger$ Proskurina et al. (2004). $\$$ Mohamed \& Yehia (2014).

2014). The $(3 \times 1)$ reconstruction rods were not observed in the GIXRD experiment. This is probably due to the $\mathrm{Ag}$ segregation that was present in the samples prepared in the GIXRD system, which inhibits the establishment of large reconstructed surface regions. The $(3 \times 1)$ superstructure was therefore neglected in the model. The most reliable film structure was obtained considering five bilayer occupancies $\left(\mathrm{Occ}_{i}\right)$ and fitting the first $A-B$ interlayer distance at the $\mathrm{Ag}$ interface $\left(d_{1}\right)$. The remaining interlayer distances were kept fixed at the value $d=c_{\text {film }} / 8=105.1 \mathrm{pm}$. In the fit, the intensities of four equi-occupied domains with equivalent structures rotated by $90^{\circ}$ were added to restore the substrate symmetry. The fit is plotted in Fig. 4 and the occupancies' bestfit values are reported in Table 3 . Their distribution corresponds to an r.m.s. roughness of about $0.4 \mathrm{~nm}$.

The most significant discrepancies between the simulated and experimental data originate from low-intensity regions close to the main peaks, where deep minima are calculated but not observed experimentally, and close to antiphase positions. This results in a relatively high $\chi^{2}$ of 7.6 . It should be emphasized, however, that most of the discrepancies probably arise from the observed $\mathrm{Ag}$ segregation at the surface. It is known that $\mathrm{Ag}$ deposited on the $\left(2^{1 / 2} \times 2^{1 / 2}\right) R 45^{\circ} \mathrm{Fe}_{3} \mathrm{O}_{4}(001)$ surface grows both on specific crystallographic sites and as clusters, depending on the annealing conditions (Bliem, Kosak et al., 2014). A fraction of an Ag monolayer located on specific sites on the surface would correspond in modulus to a few percent of the $\mathrm{CoFe}_{2} \mathrm{O}_{4}$ film's scattering amplitude at $Q=0$. While this interference can be neglected for strong peaks, it may substantially change the diffracted intensity close to minima. Nevertheless, the comparison between the crystal- lographic model and the experimental X-ray diffraction data using another figure of merit, the so-called $R$ factor, which neglects experimental errors, gives a very reasonable value of 0.17 .

$\mathrm{CoFe}_{2} \mathrm{O}_{4}$ has the same spinel structure as magnetite. In the absence of $1 / 6$ of the cations on octahedral sites in magnetite, $\mathrm{Fe}_{2} \mathrm{O}_{3}$ with the maghemite structure is obtained. For this reason, the octahedral site occupancy of the cobalt ferrite film was also checked. The resulting occupancy $\operatorname{Occ}(B)$ was found to be between 1 and 0.95 .

\section{Photoemission}

X-ray photoelectron spectra were collected ex situ on the sample grown in the STM setup. This technique allows an initial determination of the inversion parameter. Although less precise than RXD, it is available in the laboratory and allows an optimization of the growing conditions prior to synchrotron radiation experiments. The sample was first degassed for a few hours at approximately $450 \mathrm{~K}$ in UHV to remove adsorbed molecules (no trace of carbon contamination was detected by XPS).

Figs. 7(a) and 7(b) show the Co $2 p$ and Fe $2 p$ core-level photoemission regions, respectively. To analyse the spectra we closely followed the approach described by Aghavnian et al. (2015). Thus, we fitted the whole spectrum, but took into account only the $2 p_{3 / 2}$ component, because its higher intensity gives greater precision.

The binding energy depends on the valence state and on the local structural environment. The Co ions in ferrite are divalent, and a splitting is observed for atoms on octahedral ( $B$, $D_{3 d}$ symmetry) and tetrahedral ( $A, T_{d}$ symmetry) sites. Each sublevel has a shake-up satellite, due to the excitation of a $3 d$ electron by the core-level photoelectron. Therefore, the Co $2 p$ level was fitted with three doublets, after subtraction of a suitable background (a Shirley background, plus a linear one which represents the energy-loss contribution of peaks at lower binding energy). $\mathrm{Fe}$ is trivalent in $\mathrm{CoFe}_{2} \mathrm{O}_{4}$. Analysis of its $2 p$ level is difficult because of the presence of the oxygen Auger peak and due to the fact that the shake-up satellites are poorly defined. Therefore, in this case a reliable fit cannot be found without constraints.

Aghavnian et al. (2015) determined the inversion degree in $\mathrm{CoFe}_{2} \mathrm{O}_{4} / \mathrm{BaTiO}_{3}$ films by X-ray magnetic circular dichroism (XMCD) and used their results to fit XPS $2 p$ data. Here, for both elements, we constrained the energy splitting between $A$ and $B$ sites to the values given by Aghavnian et al. (2.4 and $2.65 \mathrm{eV}$ for $\mathrm{Co}$ and $\mathrm{Fe}$, respectively). The areas of the $\mathrm{Co}$ $2 p_{3 / 2}-D_{3 d}$ and Co $2 p_{3 / 2}-T_{d}$ peaks directly give the fraction of Co on octahedral sites, i.e. the inversion parameter. A value of about 0.81 is obtained. On the other hand, the equivalent analysis of the $\mathrm{Fe} 2 p_{3 / 2}$ doublet provides an estimation that $50(10) \%$ of Fe is located on tetrahedral sites. These results must be compared with the values obtained by the quantitative resonant diffraction analysis [0.88(5) and $45(3) \%$, respectively; see next section]. 


\section{Resonant X-ray diffraction}

RXD exploits the change in the atomic form factor close to an absorption edge to study the composition and atomic environment of a given crystallographic site (Grenier \& Joly, 2014). In the present experiment, the intensity changes of several ferrite film diffraction peaks were measured by scanning the energy close to both the $\mathrm{Fe}$ and $\mathrm{Co}$ absorption $K$ edges. Experimentally, this requires the diffractometer circles to move in such a way that the $(H K L)$ position is kept fixed while scanning the energy. These energy scans are shown in Fig. 8 for a set of six peaks at both edges and for five additional peaks at

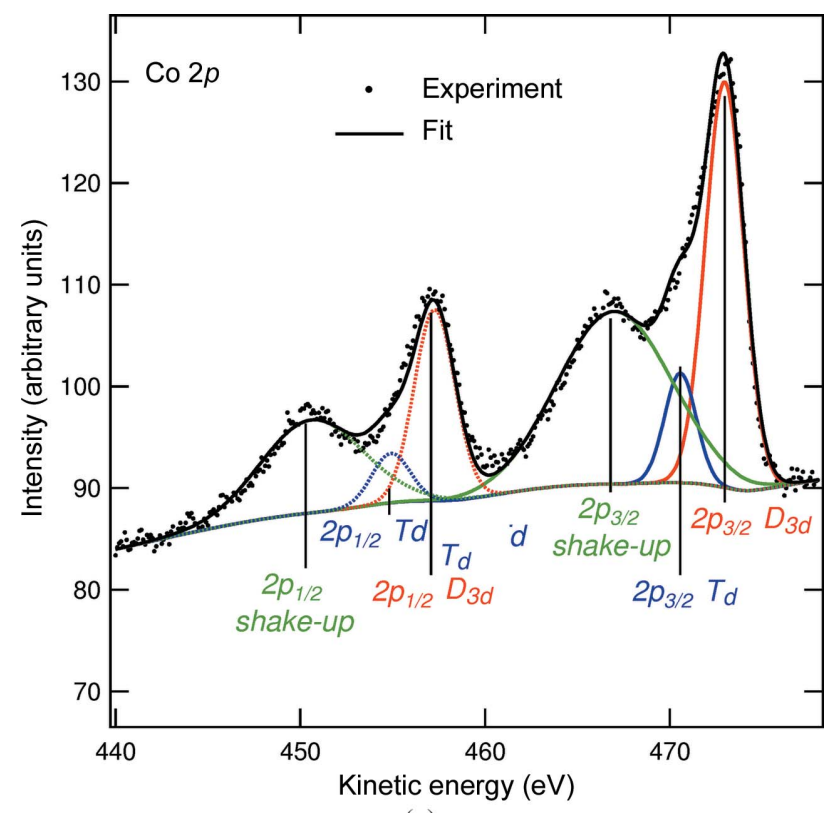

(a)

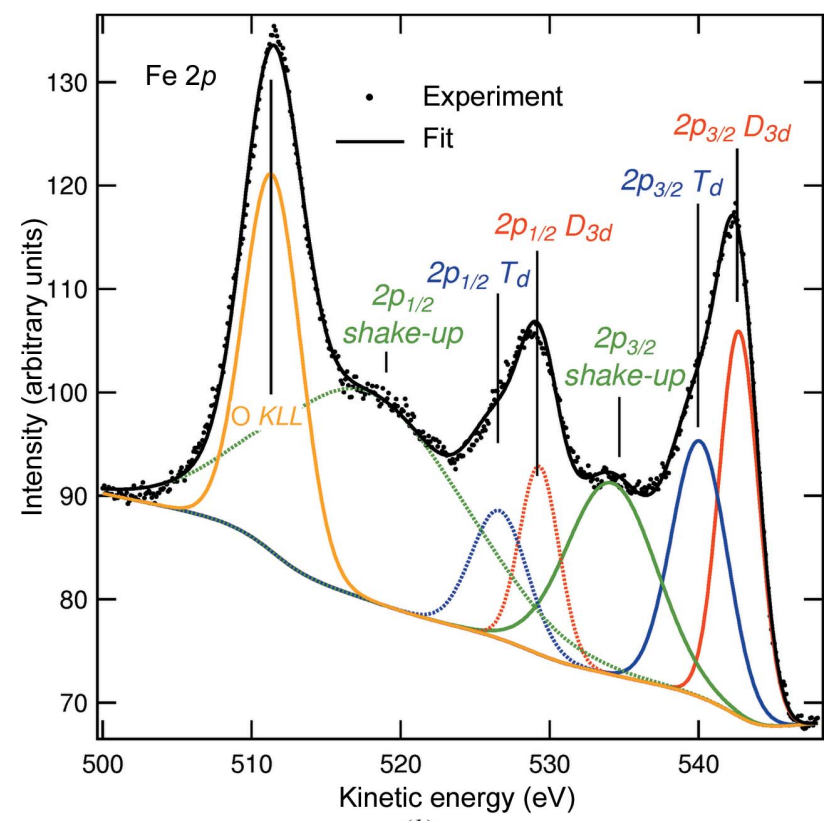

(b)

Figure 7

Experimental (a) Co and (b) Fe $2 p$ core-level photoemission lines and best fits. Each sublevel $\left(2 p_{3 / 2}\right.$ and $\left.2 p_{1 / 2}\right)$ splits into a tetrahedral component $\left(T_{d}\right)$, an octahedral one $\left(D_{3 d}\right)$ and a shake-up satellite. The oxygen $K L L$ Auger line is also observed in the Fe $2 p$ region. the Fe edge only. Some of these peaks exhibit a very strong intensity variation, which makes this technique very powerful in determining the stoichiometry of the octahedral and tetrahedral sites of the spinel structure. The $\mathrm{CoFe}_{2} \mathrm{O}_{4}$ unit-cell structure factors can be written as the sum of three contributions originating from the $A, B$ and oxygen sites:

$$
F(H K L)=F_{B}+F_{A}+F_{\mathrm{O}} .
$$

While most of the reflections are sensitive to all atomic sites, it is easy to calculate that

$$
F(2+4 n, 0,2)=(-1)^{n+1} \times 8 f_{A}+F_{\mathrm{O}},
$$

and

$$
F(2+4 n, 2,2)=16 f_{B}+F_{\mathrm{O}},
$$

where $f_{A}=f_{\mathrm{Co}} x_{A, \mathrm{Co}}+f_{\mathrm{Fe}}\left(1-x_{A, \mathrm{Co}}\right)$, and $x_{A, \mathrm{Co}}$ is the fraction of $A$ sites occupied by Co (and analogously for $f_{B}$ ).

This means that the (202) and (602) reflections plotted in Fig. 8 are sensitive to the tetrahedral cation sites only, and the (222) and (622) ones to the octahedral ones only. A first inspection of the respective curves brings the conclusion that the inversion parameter is quite high because, for example, the intensity variation of the (202) reflection is much larger at the $\mathrm{Fe}$ edge than at the Co edge, i.e. the tetrahedral site is iron rich. In the past, the order parameter of bulk cobalt ferrite was measured using the multiwavelength anomalous diffraction (MAD) technique, which measures the intensity at a few fixed energy values before the edge (Waseda et al., 1995). A more quantitative analysis requires precise knowledge of the resonant contribution to the scattering factors, which is very sensitive to the oxidation state and to the environment of the selected element. Here, this resonant contribution was calculated using FDMNES (Bunău \& Joly, 2009), an ab initio program already extensively used to simulate XANES and RXD. Its density functional theory full-potential approach makes it especially appropriate for simulating absorption edges of chemical elements embedded in non-close-packed surroundings or on low-symmetry sites. The specific $\mathrm{Co}$ and $\mathrm{Fe}$ scattering factors were calculated for atoms located on both octahedral and tetrahedral sites, taking a spinel structure with statistical occupancy of the cation sites. They were then inserted into the unit-cell structure factor to calculate the intensities. The occupancy of each site was obtained through a best fit of the experimental intensities. For this purpose we used the strong distance-reliability factors $R_{1}$ (Philip \& Rundgren, 1984) developed for the comparison of sets of spectra in LEED. Since the energy range and the number of spectra are very similar between this spectroscopy and our use of resonant X-ray diffraction, this criterion is very convenient. We found $R_{1}=0.0226$ to obtain the agreement shown in Fig. 8 (continuous red lines), giving the result that the tetrahedral and octahedral sites are occupied by Co at $13(5) \%$ and 46 (3)\%, respectively. This gives an inversion parameter of 0.88 (5) and an average stoichiometry of $\mathrm{Co}_{1.05} \mathrm{Fe}_{1.95} \mathrm{O}_{4}$. The inversion parameter is quite large compared with that obtained for bulk samples; in the MAD experiment cited above, an inversion parameter of 0.78 was found. In Fig. 8, the 
intensity calculated assuming statistical occupancy of the cation sites is also shown for comparison (dashed green lines).

\section{Summary and conclusions}

Ultrathin cobalt ferrite layers were grown on $\mathrm{Ag}(001)$ by molecular-beam epitaxy following a three-step method. The films are (001) oriented and have a sharp interface, a relatively flat surface and a bulk-like crystallographic structure. The substrate induces a slight compressive strain and the inversion parameter, determined by resonant X-ray diffraction, is close to 1 . These characteristics make such films an ideal insulating barrier for spintronic applications, particularly for room- temperature spin filtering, since the inversion parameter is linked to the height of the spin-dependent energy gap at the Fermi level (Szotek et al., 2006).

Theoretical calculations have also shown that the strain and degree of inversion are correlated; a decrease in the unit-cell constant favours the inverse configuration with respect to the normal state (Fritsch \& Ederer, 2011). Therefore, the nature of the substrate and the growth mode can have a strong influence on the cation distribution.

Both calculation (Fritsch \& Ederer, 2010) and experiment (Gao et al., 2009) agree that a compressive strain in cobalt ferrite films induces an in-plane magnetization. Such layers can then be combined with other transition metal oxides to
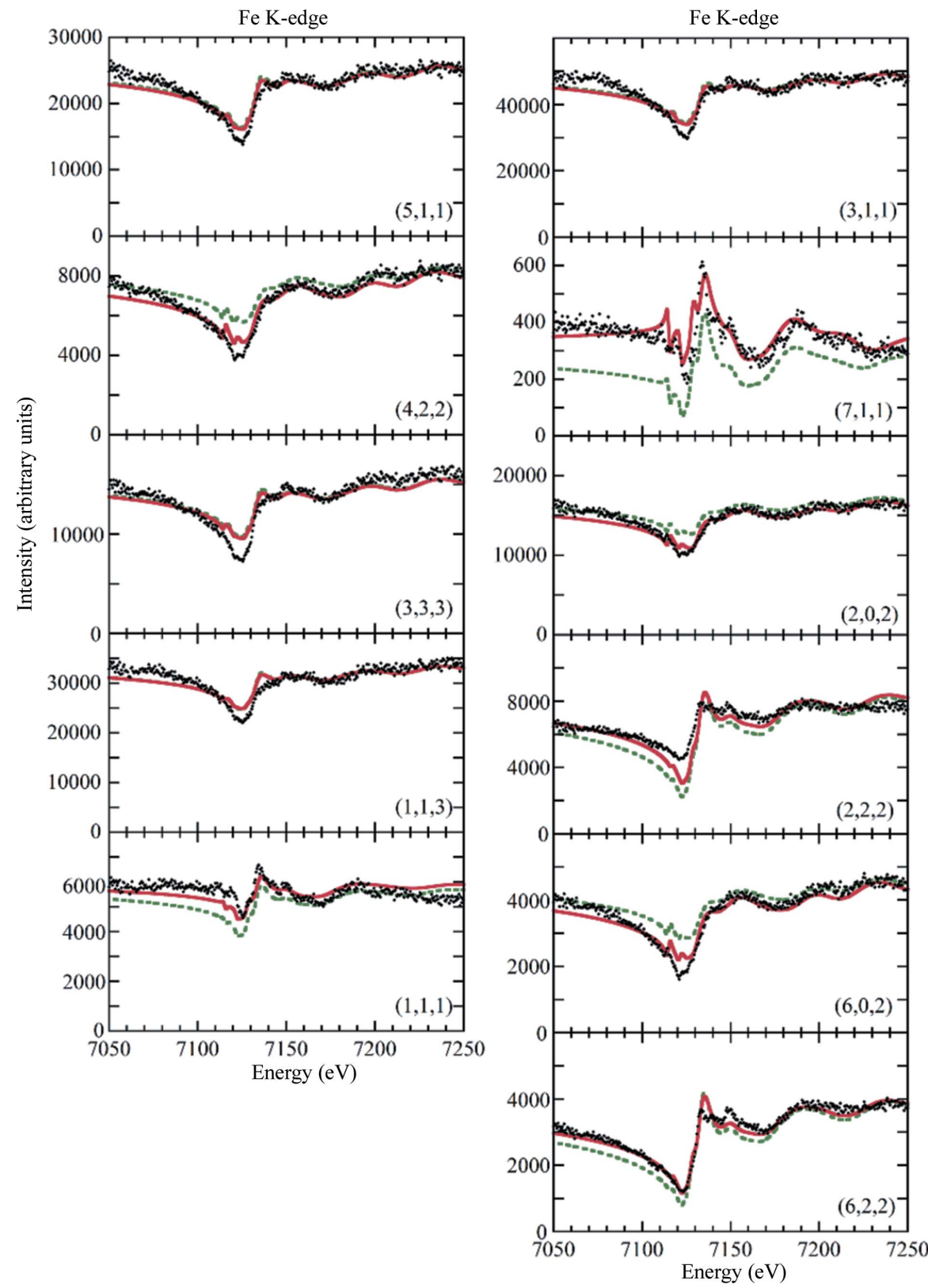

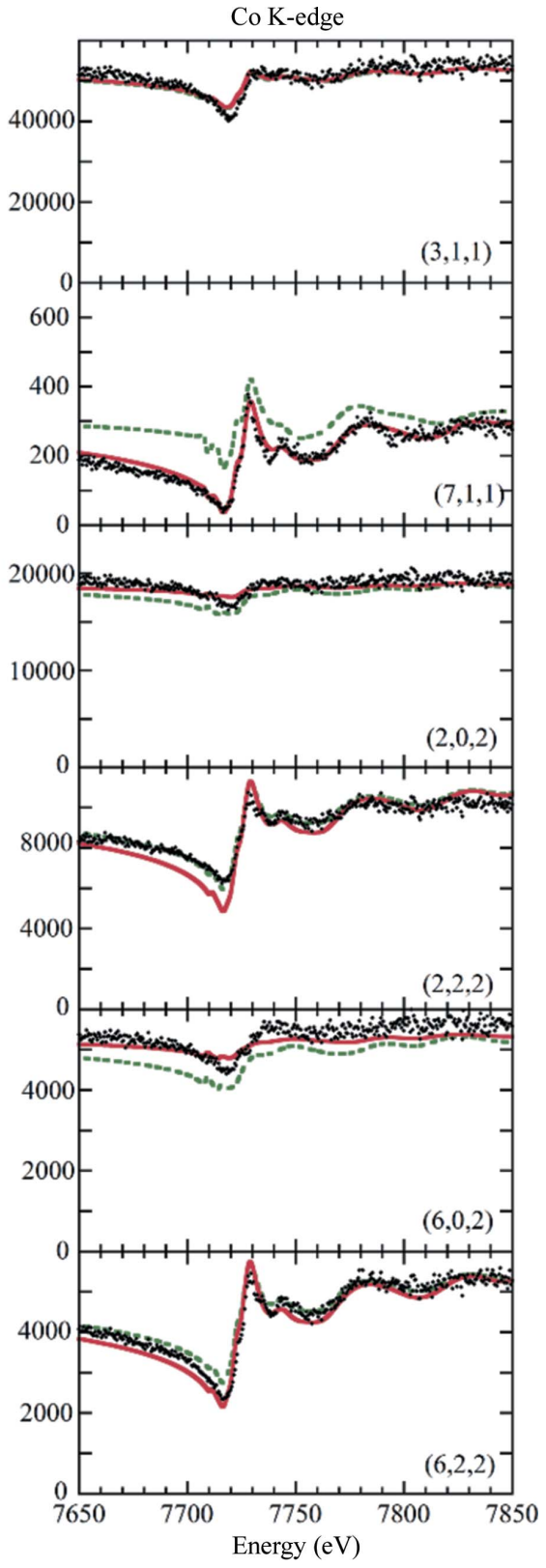

Figure 8

Experimental RXD of selected $(H K L)$ reflections at the Fe (left and central columns) and Co (right) $K$ edges (black symbols), and the calculated intensities for the best-fit occupancy (continuous red lines) and for statistical occupancy (dashed green lines). 
form model multilayered structures whose properties can be tuned and calculated theoretically. For example, cobalt ferrite, magnetite, $\mathrm{MgO}$ and other transition metal oxides can be layered up to form magnetic tunnel junctions (Chapline \& Wang, 2006) and other functional devices in coherent epitaxy. This will help to develop an understanding of the influence of the structural parameters on the physical properties.

\section{Acknowledgements}

Beam time on the French CRG BM32 beamline of the ESRF is acknowledged.

\section{Funding information}

The following funding is acknowledged: Agence Nationale de la Recherche (award No. EQUIPEX ANR-11-EQPX-0010); European Commission [project EFA194/16 TNSI (POCTEFA/UE-FEDER) to V. Langlais and X. Torrelles].

\section{References}

Aghavnian, T., Moussy, J.-B., Stanescu, D., Belkhou, R., Jedrecy, N., Magnan, H., Ohresser, P., Arrio, M.-A., Sainctavit, P. \& Barbier, A. (2015). J. Electron Spectrosc. Relat. Phenom. 202, 16-21.

Bliem, R., Kosak, R., Perneczky, L., Novotny, Z., Gamba, O., Fobes, D., Mao, Z., Schmid, M., Blaha, P., Diebold, U. \& Parkinson, G. S. (2014). ACS Nano, 8, 7531-7537.

Bliem, R., McDermott, E., Ferstl, P., Setvin, M., Gamba, O., Pavelec, J., Schneider, M. A., Schmid, M., Diebold, U., Blaha, P., Hammer, L. \& Parkinson, G. S. (2014). Science, 346, 1215-1218.

Bozorth, R. M., Tilden, E. F. \& Williams, A. J. (1955). Phys. Rev. 99, 1788-1798.

Brabers, V. A. M. (1995). Handbook of Magnetic Materials, edited by K. H. J. Buschow, pp. 189-324. Amsterdam: Elsevier.

Bunău, O. \& Joly, Y. (2009). J. Phys. Condens. Matter, 21, 345501.

Caffrey, N. M., Fritsch, D., Archer, T., Sanvito, S. \& Ederer, C. (2013). Phys. Rev. B, 87, 024419 .

Chambers, S. A., Farrow, R. F. C., Maat, S., Toney, M. F., Folks, L., Catalano, J. G., Trainor, T. P. \& Brown, G. E. Jr (2002). J. Magn. Magn. Mater. 246, 124.

Chapline, M. G. \& Wang, S. X. (2006). Phys. Rev. B, 74, 014418.

Feidenhans'l, R. (1989). Surf. Sci. Rep. 10, 105-188.

Fritsch, D. \& Ederer, C. (2010). Phys. Rev. B, 82, 104117.

Fritsch, D. \& Ederer, C. (2011). Appl. Phys. Lett. 99, 081916.
Gao, X. S., Bao, D. H., Birajdar, B., Habisreuther, T., Mattheis, R., Schubert, M. A., Alexe, M. \& Hesse, D. (2009). J. Phys. D Appl. Phys. 42, 175006.

Grenier, S. \& Joly, Y. (2014). J. Phys. Conf. Ser. 519, 012001.

Gubo, M., Ebensperger, C., Meyer, W., Hammer, L., Heinz, K., Mittendorfer, F. \& Redinger, J. (2012). Phys. Rev. Lett. 108, 066101.

Lamirand, A. D., Grenier, S., Langlais, V., Ramos, A. Y., Tolentino, H. C. N., Torrelles, X. \& De Santis, M. (2016). Surf. Sci. 647, 33-38. Lisfi, A. \& Williams, C. M. (2003). J. Appl. Phys. 93, 8143-8145.

Matzen, S., Moussy, J.-B., Mattana, R., Petroff, F., Gatel, C., WarotFonrose, B., Cezar, J. C., Barbier, A., Arrio, M.-A. \& Sainctavit, P. (2011). Appl. Phys. Lett. 99, 052514.

Meyer, W., Hock, D., Biedermann, K., Gubo, M., Müller, S., Hammer, L. \& Heinz, K. (2008). Phys. Rev. Lett. 101, 016103.

Meyerheim, H. L., Pflanz, S., Schuster, R. \& Robinson, I. K. (1997). Z. Kristallogr. 212, 327.

Mohamed, M. B. \& Yehia, M. (2014). J. Alloys Compd. 615, 181187.

Moodera, J. S., Hao, X., Gibson, G. A. \& Meservey, R. (1988). Phys. Rev. Lett. 61, 637-640.

Pentcheva, R., Wendler, F., Meyerheim, H. L., Moritz, W., Jedrecy, N. \& Scheffler, M. (2005). Phys. Rev. Lett. 94, 126101.

Philip, J. \& Rundgren, J. (1984). In Proceedings of the Conference on Determination of Surface Structure by LEED, Yorktown Heights, 1980, edited by P. M. Marcus. New York: Plenum.

Prévot, G., Coati, A., Croset, B. \& Garreau, Y. (2007). J. Appl. Cryst. 40, 874-882.

Proskurina, N. V., Cherepanov, V. A., Golynets, O. S. \& Voronin, V. I. (2004). Inorg. Mater. 40, 955-959.

Robinson, I. K. (1986). Phys. Rev. B, 33, 3830-3836.

Robinson, I. K. (1991). Handbook on Synchrotron Radiation, edited by G. S. Brown \& D. E. Moncton, Vol. 3, p. 221. Amsterdam: NorthHolland.

Stanka, B., Hebenstreit, W., Diebold, U. \& Chambers, S. A. (2000). Surf. Sci. 448, 49-63.

Szotek, Z., Temmerman, W. M., Ködderitzsch, D., Svane, A., Petit, L. \& Winter, H. (2006). Phys. Rev. B, 74, 174431.

Torelli, P., Soares, E. A., Renaud, G., Valeri, S., Guo, X. X. \& Luches, P. (2007). Surf. Sci. 601, 2651-2655.

Vlieg, E. (1997). J. Appl. Cryst. 30, 532-543.

Voogt, F. C., Palstra, T. T. M., Niesen, L., Rogojanu, O. C., James, M. A. \& Hibma, T. (1998). Phys. Rev. B, 57, R8107-R8110.

Waseda, Y., Shinoda, K. \& Sugiyama, K. (1995). Z. Naturforsch. 50a, 1199.

Yanagihara, H., Uwabo, K., Minagawa, M., Kita, E. \& Hirota, N. (2011). J. Appl. Phys. 109, 07C122.

Zavaliche, F., Zheng, H., Mohaddes-Ardabili, L., Yang, S. Y., Zhan, Q., Shafer, P., Reilly, E., Chopdekar, R., Jia, Y., Wright, P., Schlom, D. G., Suzuki, Y. \& Ramesh, R. (2005). Nano Lett. 5, 1793-1796. 